\title{
Weak interactions of COVID-19 virus Mpro and peptide-like inhibitor N3: revealing the role of non- conventional hydrogen bonds by theoretical methods
}

\section{Ponciano García-Gutiérrez}

Universidad Autónoma Metropolitana-Iztapalapa

Rafael A. Zubillaga

Universidad Autónoma Metropolitana-Iztapalapa

llich A. Ibarra

Universidad Nacional Autónoma de México

Ana Martínez

Universidad Nacional Autónoma de México

Rubicelia Vargas ( $\square$ ruvf@xanum.uam.mx)

Universidad Autónoma Metropolitana-Iztapalapa

Jorge Garza ( $\nabla$ jgo@xanum.uam.mx )

Universidad Autónoma Metropolitana-Iztapalapa

\section{Research Article}

Keywords: COVID-19, Non-covalent interactions, Non-conventional hydrogen bonds, DFT, Atoms in molecules

Posted Date: May 29th, 2020

DOI: https://doi.org/10.21203/rs.3.rs-31850/v1

License: (c) (1) This work is licensed under a Creative Commons Attribution 4.0 International License. Read Full License 


\title{
Weak interactions of COVID-19 virus $M^{\text {pro }}$ and peptide-like inhibitor N3: revealing the role of non-conventional hydrogen bonds by theoretical methods
}

\author{
Ponciano García-Gutiérrez ${ }^{1}$, Rafael A. Zubillaga ${ }^{1}$, Ilich A. Ibarra² ${ }^{2}$ Ana Martínez², \\ Rubicelia Vargas ${ }^{1, *}$, and Jorge Garza ${ }^{1, *}$
}

\author{
${ }^{1}$ Departamento de Química, División de Ciencias Básicas e Ingeniería, Universidad Autónoma \\ Metropolitana-Iztapalapa, San Rafael Atlixco 186, Col. Vicentina, Iztapalapa. AP Postal 55-534, CP 09340, CDMX, \\ México. \\ ${ }^{2}$ Instituto de Investigaciones en Materiales, Universidad Nacional Autónoma de México, Circuito Exterior S. N., \\ Ciudad Universitaria, CP 04510, CDMX, México. \\ *ruvf@xanum.uam.mx, jgo@xanum.uam.mx
}

\begin{abstract}
Two models consisting of 469 atoms each one, for the interaction between the N3 inhibitor and the main protease of SARS-CoV (SARS-CoV $\mathrm{M}^{\text {pro }}$ ) and SARS-CoV-2 (SARS-CoV-2 $\mathrm{M}^{\text {pro }}$ ) viruses, were used to reveal by quantum chemistry methods the non-covalent interactions involved in these systems. Through the Density Functional Theory and the Quantum Theory of Atoms in Molecules the main conclusion reached by this study indicates that $\mathrm{C}-\mathrm{H} \ldots \mathrm{O}$ and $\mathrm{C}-\mathrm{H} \ldots \mathrm{H}$ hydrogen bonds are crucial to describe attractive interactions in these complexes. In general, these contacts are overlooked in many studies. However, these non-conventional hydrogen bonds represent more than a half of the energy interaction estimated for non-covalent contacts. These results are quite important for the design of new drugs since these interactions could drive the action mechanisms against these viruses. Hydrogen bonds are crucial to describe correctly the interactions between inhibitors and the main proteases.
\end{abstract}

\section{Introduction}

The beginning of a new decade, in many cultures, represents a positive and extremely exciting event. The year 2020 should have been a very stimulating year for our diverse and multicultural society, around the world, due to a wide range of extremely anticipated and prestigious activities (e.g., the Olympics in Tokyo 2020). However, 4 months into 2020 and our planet is sadly facing the greatest test since World War II: an existential global health crisis through the outbreak of a novel coronavirus respiratory disease 2019 (COVID-19) caused by SARS-CoV-2 virus. An interesting contribution by Menachery et al. ${ }^{1}$ published in 2015, almost prophetically, warned us about "bat coronaviruses shows potential for human emergence". The suggestion that COVID-19 may have originated in Chinese bat populations will lead to antagonic, scientific and political, conclusions. However, regardless where COVID-19 is actually coming from, this pandemic has been taking human lives worldwide. In addition, our global economy is transiting into a huge recession as businesses close and millions of jobs are lost (as a direct result of globalization and interconnected international economies). Governments around the world have reacted fragmentary and in unambiguously different ways. Extensive spread of COVID-19 in each continent, this virus has a higher transmission rate than SARS-CoV which emerged in $2002,{ }^{2}$ is now demonstrating how serious this treat is to life. Thus, believing that this pandemic can be solved by national governments only operating individually, it is frankly a daring unreality. Global leaders, not only G20 leaders, must rise a strong and common battle-front to defeat this pandemic. This of course, is not an exclusive task for the international governments. Among the different strategic actions (coming from different sector of society) to achieve such interactive collaborative role, the scientific community must rapidly cooperate across borders to try to understand the virus and develop new methods to combat it. Therefore, it is of vital relevance that clinicians, epidemiologist and basic researchers (i.e., via the implementation of computational resources) all work together to obtain, analyze and interpret meaningful data related to COVID-19. Most importantly, these scientific contributions must be available as soon as possible to provide valuable insights on the collective fight against COVID-19, perhaps the greatest threat in human history.

Family Coronavirida $e^{3}$ is constructed from enveloped viruses with a positive-strand RNA genome. These coronaviruses 
mainly contain four structural proteins: spike protein, envelope protein, membrane protein, and nucleocapsid protein ${ }^{4}$. Spike proteins are relevant during the virus infection since they promote host attachment and virus-cell membrane fusion. SARS$\mathrm{CoV}-2$ is a coronavirus that belongs to this family. Several compounds have been investigated in order to find antiviral drugs or a vaccine against human COVID-19 infection ${ }^{5}$, but an effective and unquestionable antiviral strategy against this emergency is not yet available. One possible target is to find an inhibitor of the SARS-CoV-2 $\mathrm{M}^{\text {pro }}$ and for this reason there are several investigations in this direction. SARS-CoV-2 is a new coronavirus but there are others. In particular SARS-CoV is a well-studied coronavirus of which have been reported inhibitors that might help us to control the infections. In 2016, Wang et al. reported the crystal structure of a synthetic peptidomimetic inhibitor named N3 forming a complex with SARS-CoV M ${ }^{\text {pro3 }}$. From the comparison with different proteases of other coronaviruses, they demonstrated that the $\mathrm{M}^{\text {pro }}$ is a conserved drug target in coronaviruses ${ }^{3}$. Yang et al. ${ }^{6}$ reported that all $\mathrm{CoV}$ main proteases have a highly conserved substrate-recognition pocket. The X-ray crystal structure of SARS-CoV-2 $\mathrm{M}^{\text {pro }}$ was published in February $2020^{5}$. It has a dimer-like structure that present $96 \%$ sequence homology with that of SARS-CoV $\mathrm{M}^{\mathrm{pr} 7,8}$. This is certainly important to the design of novel inhibitors since this similarity allows us to think that it is possible to develop inhibitors against SARS-CoV-2 $\mathrm{M}^{\text {pro }}$ using the information that we already have for SARS-CoV $\mathrm{M}^{\text {pro }}$.

Peptide-like inhibitor N3 (Figure 1) has been investigated with SARS-CoV $\mathrm{M}^{\text {pro }}$ and also with SARS-CoV-2 $\mathrm{M}^{\text {pro }}$. The crystal structure of N3 with SARS-CoV-2 $\mathrm{M}^{\text {pro }}$ is already published ${ }^{5}$. Authors report with detail the specific interactions of N3 with the main protease and they compared with the N3-SARS-CoV $\mathrm{M}^{\text {pro }}$ complex. Authors conclude that inhibitors bind to the main proteases in a similar mode and therefore, the action mechanisms might be similar. However, these are X-Ray structural data and with these results it is not possible to give a full characterization of molecular interactions. To understand the action mechanism it is important to have accurate information on the inhibitor-protein interactions. One of the efforts to have this information is the fragment molecular orbital-based interaction analysis that has been reported previously; the authors analyzed the complex formed with the SARS-CoV-2 $\mathrm{M}^{\text {pro }}$ and the peptide-like inhibitor N3. This description is partial as they used fragment molecular orbitals ${ }^{9}$. It is crucial to have accurate information of the interaction of SARS-CoV-2 $\mathrm{M}^{\text {pro }}$ and the N3 inhibitor and for this reason, in this investigation we determine and compare all the interactions, including weak interactions, of N3-SARS-CoV M $\mathrm{M}^{\text {pro }}$ and N3-SARS-CoV-2 $\mathrm{M}^{\text {pro }}$ complexes. Hydrogen bonds, that are crucial for the description of the interactions and activity of these compounds, are well characterized in this work using the Quantum Theory of Atoms in Molecules (QTAIM) ${ }^{10}$ approach. From this theoretical analysis, we determine the differences between N3-SARS-CoV $\mathrm{M}^{\text {pro }}$ and N3-SARS-CoV-2 $\mathrm{M}^{\text {pro }}$ complexes. These results considerably will contribute to the development of effective drugs against COVID-19.

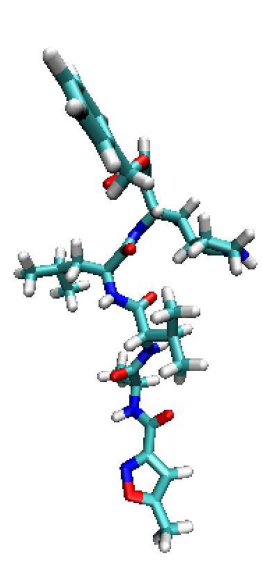

(I)

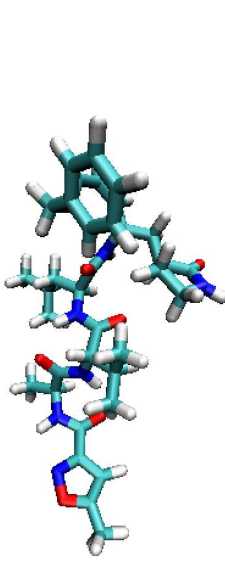

(II)

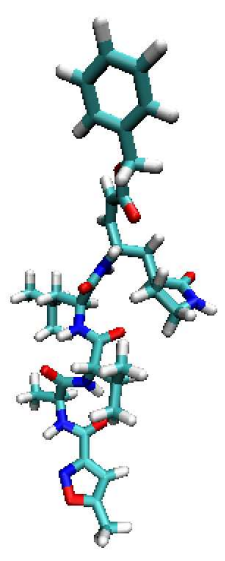

(III)

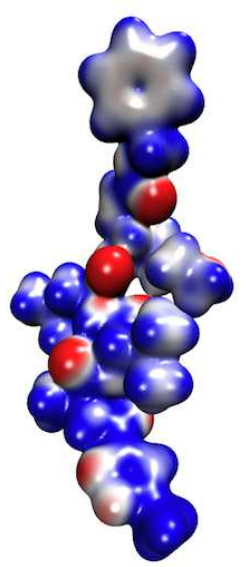

(MEP of III)

Figure 1. Three rotamers of the N3 inhibitor. The Molecular Electrostatic Potential (MEP) of the rotamer (III) is depicted on the right side as an example. Blue regions indicate positive values and red zones indicate negative values.

\section{Results and discussion}

In this article, two models were used to describe the interaction between N3 and the main proteases of SARS-CoV and SARS-CoV-2. Both models were obtained from the X-ray structures (PDB ID's 2AMQ and 6Lu7, respectively) ${ }^{5,6}$ and contain 469 atoms, 97 related to the inhibitor and the remained related to the corresponding main protease. More details of the used methodology are explained in the methods section . 
The N3 inhibitor is a molecule that exhibits different rotamers. Figure 1 reports three of them. Rotamer (I) corresponds to

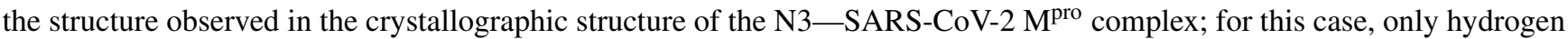
atoms positions were relaxed. The other two structures in Figure 1 (II and III) were obtained with our computational methods by relaxing completely all atoms positions. These three structures are reported in the electronic supporting information (ESI). We note that structures II and III are quite different from each other, but the energy difference between them is quite small (2.4 $\mathrm{kcal} / \mathrm{mol}$ ) and therefore it is possible to say that this inhibitor has associated conformers with similar energy. One important characteristic of this molecule is the number of lone pairs given by oxygen and nitrogen atoms involved in its structure, which give a particular feature of the Molecular Electrostatic Potential (MEP), as can be seen on the right side of Figure 1. Therefore, this molecule provides several positive and negative regions of the MEP that induce possible contacts with coronaviruses $\mathrm{M}^{\text {pro }}$ that are driven by electrostatic effects. By analyzing both complexes, N3-SARS-CoV M $^{\text {pro }}$ and N3-COVID-19 M ${ }^{\text {pro }}$, the

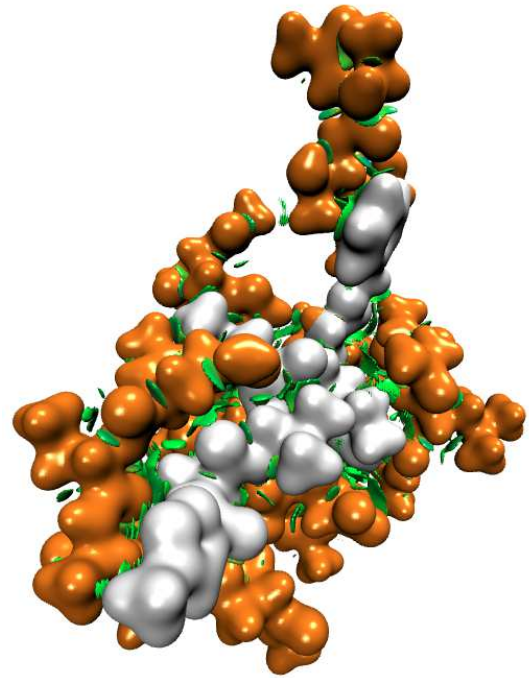

(a)

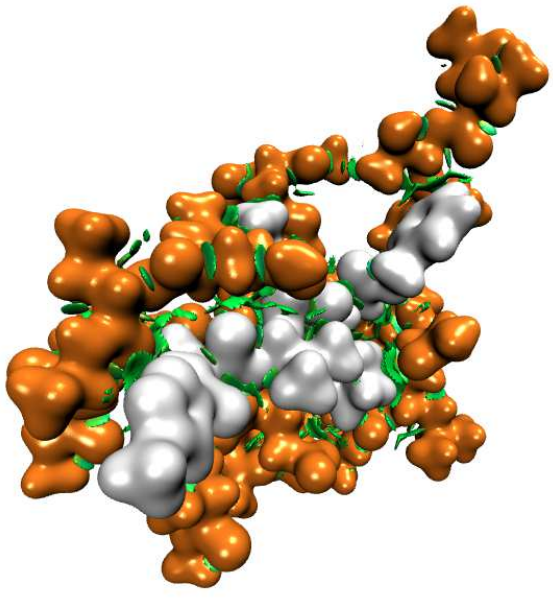

(c)

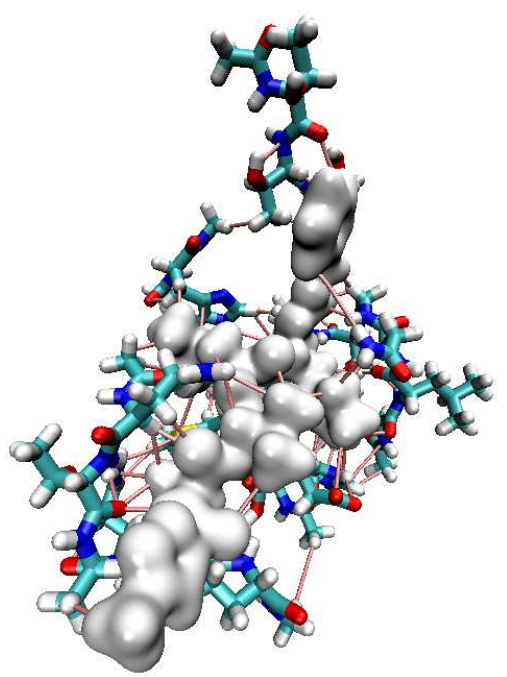

(b)



(d)

Figure 2. Isosurfaces of the electron density (orange for protase and white for inhibitor) and non-colvalent interaction index

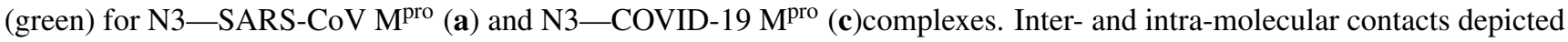
through bond paths (pinks) provided by the QTAIM for N3-SARS-Cov M ${ }^{\text {pro }}$ (b) and N3-SARS-CoV-2 M ${ }^{\text {pro }}$ (d) systems.

non-covalent interaction index $(\mathrm{NCI})^{11}$ reveals weak non-covalent interactions for both molecular systems, as we can see from Figure 2. In this figure, the NCI is represented in terms of an isosurface colored mainly by green color, which indicates weak interactions (van der Waals and hydrogen bonds) between N3 and the main protease, but also intramolecular interactions. Such an isosurface forms a boundary between the electron densities delivered by our protease models and the N3 inhibitor. Without a doubt there are many non-covalent interactions between these systems.

From results delivered by the NCI, we do search all possible critical points of the electron density to characterize these 
contacts through the QTAIM approach. Thus, we performed this search of both complexes and we counted each interaction between N3 and both proteases models by looking for all bond critical points (BCP) defined within the QTAIM. From these points we found the corresponding bond paths to establish the atoms involved in each contact and with this information we determine the molecular graph of each modeled complex. We would like to emphasize that this task for systems with the number of atoms considered in this article is a remarkable challenge for common software used currently. For this reason we used the implementations proposed in the GPUAM ${ }^{12}$ project to obtain responses in reasonable times. In Figures $2 \mathbf{b}$ and $2 \mathbf{d}$ we present the bond paths (in pink color) for both complexes considered in this article. From here, we characterize each contact between the N3 and SARS-CoV-2 $\mathrm{M}^{\text {pro }}$, and SARS-CoV $\mathrm{M}^{\text {pro }}$. Table 1 is a summary of the QTAIM analysis performed to obtain the hydrogen bonds related to the interaction between N3 and $\mathrm{M}^{\text {pro }}$ from SARS-CoV and SARS-CoV-2.

Table 1. Hydrogen bonds obtained for N3-SARS-CoV $M^{\text {pro }}$ and N3-SARS-CoV-2 $\mathrm{M}^{\text {pro }}$ models. $\mathrm{d}(\mathrm{A} \cdots \mathrm{H})$ represents the

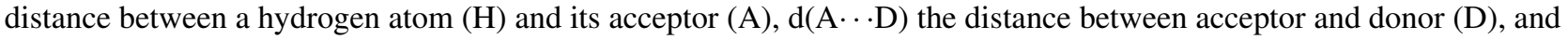
$\varangle \mathrm{A} \cdots \mathrm{H}-\mathrm{D}$ the angle involved in a hydrogen bond. The electron density evaluated at a critical point corresponding to a hydrogen bond is represented by $\rho(\mathrm{BCP})$.

\begin{tabular}{|c|c|c|c|c|c|c|c|c|c|c|}
\hline \multirow[t]{2}{*}{ Hydrogen bond } & \multicolumn{5}{|c|}{ N3-SARS-CoV M ${ }^{\text {pro }}$} & \multicolumn{5}{|c|}{ N3-SARS-CoV-2 Mpro } \\
\hline & \# & $\mathrm{d}(\mathrm{A}-\mathrm{H})$ & $\mathrm{d}(\mathrm{A}-\mathrm{D})$ & $\varangle \mathrm{A}-\mathrm{H}-\mathrm{D}$ & $\rho(\mathrm{BCP})$ & \# & $\mathrm{d}(\mathrm{A}-\mathrm{H})$ & $\mathrm{d}(\mathrm{A}-\mathrm{D})$ & $\varangle \mathrm{A}-\mathrm{H}-\mathrm{D}$ & $\rho(\mathrm{BCP})$ \\
\hline \multirow{3}{*}{$\mathrm{N}-\mathrm{H} \cdots \mathrm{O}$} & \multirow{3}{*}{7} & 1.615 & 2.639 & 104.5 & 0.004 & \multirow{3}{*}{8} & 1.795 & 2.803 & 149.8 & 0.004 \\
\hline & & 2.162 & 3.026 & 150.7 & 0.022 & & 2.073 & 3.054 & 163.1 & 0.023 \\
\hline & & 2.763 & 3.529 & 173.6 & 0.054 & & 2.815 & 3.724 & 172.7 & 0.034 \\
\hline \multirow{3}{*}{$\mathrm{C}-\mathrm{H} \cdots \mathrm{O}$} & \multirow{3}{*}{25} & 1.516 & 2.508 & 113.1 & 0.001 & \multirow{3}{*}{27} & 2.124 & 2.622 & 104.3 & 0.001 \\
\hline & & 2.563 & 3.389 & 134.8 & 0.013 & & 2.740 & 3.520 & 131.0 & 0.007 \\
\hline & & 3.509 & 4.346 & 154.3 & 0.069 & & 3.339 & 4.096 & 168.2 & 0.024 \\
\hline \multirow{3}{*}{$\mathrm{C}-\mathrm{H} \cdots \mathrm{H}$} & \multirow{3}{*}{15} & 1.918 & 2.343 & 98.7 & 0.003 & \multirow{3}{*}{15} & 1.554 & 2.242 & 102.5 & 0.002 \\
\hline & & 2.274 & 3.132 & 137.9 & 0.007 & & 2.249 & 3.073 & 134.9 & 0.009 \\
\hline & & 2.755 & 3.714 & 174.6 & 0.014 & & 2.993 & 3.889 & 170.7 & 0.029 \\
\hline \multirow{3}{*}{$\mathrm{C}-\mathrm{H} \cdots \mathrm{C}$} & \multirow{3}{*}{6} & 2.402 & 2.868 & 103.4 & 0.003 & \multirow{3}{*}{0} & - & - & - & - \\
\hline & & 2.671 & 3.490 & 136.3 & 0.010 & & - & - & - & - \\
\hline & & 3.158 & 4.215 & 162.3 & 0.016 & & - & - & - & - \\
\hline \multirow{3}{*}{$\mathrm{C}-\mathrm{H} \cdots \mathrm{N}$} & \multirow{3}{*}{5} & 2.407 & 3.365 & 108.0 & 0.002 & \multirow{3}{*}{8} & 2.533 & 3.310 & 117.8 & 0.002 \\
\hline & & 3.192 & 3.965 & 133.0 & 0.004 & & 2.860 & 3.701 & 134.9 & 0.007 \\
\hline & & 3.461 & 4.396 & 168.0 & 0.014 & & 3.591 & 4.420 & 153.2 & 0.011 \\
\hline \multirow{3}{*}{$\mathrm{C}-\mathrm{H} \cdots \mathrm{S}$} & \multirow{3}{*}{2} & 3.576 & 4.118 & 112.4 & 0.002 & \multirow{3}{*}{1} & \multirow{3}{*}{2.977} & \multirow{3}{*}{3.949} & \multirow{3}{*}{148.6} & \multirow{3}{*}{0.007} \\
\hline & & 3.647 & 4.416 & 132.5 & 0.002 & & & & & \\
\hline & & 3.718 & 4.714 & 152.5 & 0.003 & & & & & \\
\hline $\mathrm{N}-\mathrm{H} \cdots \mathrm{H}$ & 1 & 2.134 & 2.677 & 111.3 & 0.009 & 0 & - & - & - & - \\
\hline $\mathrm{N}-\mathrm{H} \cdots \mathrm{N}$ & 1 & 3.211 & 3.976 & 133.7 & 0.002 & 0 & - & - & - & - \\
\hline
\end{tabular}

The QTAIM approach delivers an impressive number of hydrogen bonds in N3-SARS-CoV M ${ }^{\text {pro }}$ and N3-SARS-CoV-2 $\mathrm{M}^{\mathrm{pro}}$ models, which are recorder in Table 1. In this table there are several properties that characterize the hydrogen bonds: i) distance between hydrogen atom (H) and its acceptor (A), ii) distance between acceptor and donor (D), iii) angle $\varangle$ A $\cdots H$-D defined by the three atoms involved in a hydrogen bond, iv) electron density evaluated at the $\mathrm{BCP}, \rho(\mathrm{BCP})$, corresponding to the hydrogen bond. For each property we are reporting the lowest value (top), the average (middle and bold font) and the highest value (bottom); more specific information of hydrogen bonds of these systems is given in ESI. It is important to mention that we have considered only those contacts that exhibit $\rho(\mathrm{BCP}) \geq 0.001$ atomic units (au). From the data reported in this table, clearly $\mathrm{N}-\mathrm{H} \cdots \mathrm{O}, \mathrm{C}-\mathrm{H} \cdots \mathrm{O}$ and $\mathrm{C}-\mathrm{H} \cdots \mathrm{H}$ hydrogen bonds have an important presence in these complexes. $\mathrm{N}-\mathrm{H} \cdots \mathrm{O}$ hydrogen bonds exhibit geometrical parameters which induce appropriately this interaction, with relatively small A $\cdots \mathrm{H}$ and A $\cdots \mathrm{D}$ distances, and high $\varangle \mathrm{A} \cdots \mathrm{H}-\mathrm{D}$ angle. It is worth to mention that this hydrogen bond seems stronger in the N3-SARS-CoV-2 $\mathrm{M}^{\text {pro }}$ than that observed for N3-SARS-CoV $\mathrm{M}^{\text {pro }}$, by the values presented in Table 1. It is also important to mention that there are eight of these contacts in the N3-SARS-CoV-2 $\mathrm{M}^{\text {pro }}$ complex and seven in the N3-SARS-CoV $\mathrm{M}^{\text {pro }}$.

The $\mathrm{C}-\mathrm{H} \cdots \mathrm{O}$ contact has been recognized as a weak hydrogen bond, with high relevance to stabilize some systems ${ }^{13}$. In the two complexes considered in this article, it is clear the importance of these interactions since there are 25 contacts in N3-SARS-CoV M $\mathrm{M}^{\text {pro }}$ and 27 in N3-SARS-CoV-2 $\mathrm{M}^{\text {pro }}$. However, we cannot overlook the role of the C-H $\cdots H$ contact 
(dihydrogen bond ${ }^{14}$ ) since it appears several times in each complex; in fact, from Table 1 we observe more favorable geometrical parameters in this bond than those found for $\mathrm{C}-\mathrm{H} \cdots \mathrm{O}$ interactions. $\mathrm{C}-\mathrm{H} \cdots \mathrm{N}$ and $\mathrm{C}-\mathrm{H} \cdots \mathrm{S}$ hydrogen bonds exhibit similar electron density at the $\mathrm{BCP}$ than those observed for $\mathrm{C}-\mathrm{H} \cdots \mathrm{O}$ and $\mathrm{C}-\mathrm{H} \cdots \mathrm{H}$ interactions, although they have less occurrence in both complexes. $\mathrm{C}-\mathrm{H} \cdots \mathrm{C}, \mathrm{N}-\mathrm{H} \cdots \mathrm{H}, \mathrm{N}-\mathrm{H} \cdots \mathrm{N}$ appear only in the $\mathrm{N} 3-\mathrm{SARS}-\mathrm{CoV} \mathrm{M}^{\text {pro }}$ complex, but they are irrelevant in the stabilization of this complex. For non-covalent interactions we use the Espinosa-Molins-Lecomte (EML) approach ${ }^{15}$ to estimate the energy involved in each hydrogen bond. This approximation is quite useful to compare binding energies between two similar systems. From this analysis we found a total hydrogen bond energy of $-162.1 \mathrm{kcal} / \mathrm{mol}$ for the N3-SARS-CoV $\mathrm{M}^{\text {pro }}$ complex and $-120.9 \mathrm{kcal} / \mathrm{mol}$ for the N3-SARS-CoV-2 $\mathrm{M}^{\text {pro }}$. Thus, the energetic contribution of these interactions is $34.1 \%$ higher in the complex of $\mathrm{N} 3$ with $\mathrm{M}^{\text {pro }}$ from SARS-CoV than that observed with SARS-CoV-2. It is important to mention that this estimation of the hydrogen bond energy is useful only to compare both systems and it is not intended to estimate thermodynamic binding properties.

In terms of this energy analysis, the contribution (in percent) of each hydrogen bond is reported in Table 2. From the EML approximation, $\mathrm{N}-\mathrm{H} \cdots \mathrm{O}, \mathrm{C}-\mathrm{H} \cdots \mathrm{O}$ and $\mathrm{C}-\mathrm{H} \cdots \mathrm{H}$ hydrogen bonds contain the major percent of the total energy. For the N3-SARS-CoV M $\mathrm{M}^{\text {pro }}$ complex, the $\mathrm{C}-\mathrm{H} \cdots \mathrm{O}$ contact contributes with more than a half of the total energy; the C-H $\cdots \mathrm{H}$ contact has also an important contribution to the total energy. From this analysis we conclude that $\mathrm{C}-\mathrm{H} \cdots \mathrm{O}$ and $\mathrm{C}-\mathrm{H} \cdots \mathrm{H}$ hydrogen bonds contribute with an important percent to the energy contribution of non-covalent interactions. If we consider only the $\mathrm{N}-\mathrm{H} \cdots \mathrm{O}$ hydrogen bond as main contribution to the stabilization of these systems by the side of non-covalent interactions then we are making a big mistake, since most of the energy stabilization is coming from the other hydrogen bonds.

Table 2. Energy contribution, in percent, of each hydrogen bond type found in N3-SARS-CoV $M^{\text {pro }}$ and N3-SARS-CoV-2 $\mathrm{M}^{\text {pro }}$ complexes.

\begin{tabular}{|c|c|c|c|c|c|c|c|c|}
\hline Modeled complex & \multicolumn{8}{|c|}{ Hydrogen bond type } \\
\hline & $\mathrm{N}-\mathrm{H} \cdots \mathrm{O}$ & $\mathrm{C}-\mathrm{H} \cdots \mathrm{O}$ & $\mathrm{C}-\mathrm{H} \cdots \mathrm{H}$ & $\mathrm{C}-\mathrm{H} \cdots \mathrm{C}$ & $\mathrm{C}-\mathrm{H} \cdots \mathrm{N}$ & $\mathrm{C}-\mathrm{H} \cdots \mathrm{S}$ & $\mathrm{N}-\mathrm{H} \cdots \mathrm{H}$ & $\mathrm{N}-\mathrm{H} \cdots \mathrm{N}$ \\
\hline N3-SARS-CoV M ${ }^{\text {pro }}$ & 25.8 & 53.3 & 10.4 & 6.9 & 2.2 & 0.3 & 0.9 & 0.2 \\
\hline N3-SARS-CoV-2 $M^{\text {pro }}$ & 37.4 & 34.1 & 19.9 & 0.0 & 7.8 & 0.8 & 0.0 & 0.0 \\
\hline
\end{tabular}

The positions of hydrogen bonds predicted by our computational methodology is an important contribution since we comprehensively described all the hydrogen bonds involved in the inhibitor-protease interaction. Other methodologies to describe inhibitor-protease interactions are based on molecular mechanics that used parametrizations for the description of the hydrogen bonds. We are stressing that in N3-SARS-CoV M ${ }^{\text {pro }}$ and N3-SARS-CoV-2 $\mathrm{M}^{\text {pro }}$ complexes, there are many non-conventional hydrogen bonds that must be considered in such parametrization; as a matter of fact, $\mathrm{C}-\mathrm{H} \cdots \mathrm{O}$ unconventional hydrogen bonds contribute as much or more than conventional N-H $\cdots$ O hydrogen bonds, as can be seen in Table 2 . How important are the hydrogen bonds found in this study? We have designed two models to study the relevance of hydrogen bonds

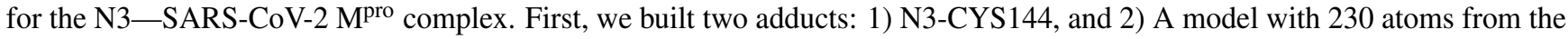
N3-SARS-CoV-2 Mpro complex. In both cases there is the C-S covalent bond involved in the N3 and the main protease. Both structures were fully optimized (reported in ESI) and measured the two C-S bond lengths where the sulfur atom is involved. For the N3-CYS144 adduct we obtained $1.832 \AA$ and $1.818 \AA$, and for the second model $1.835 \AA$ and $1.809 \AA$. For the N3-SARS-CoV-2 M pro complex, from the single crystal X-ray diffraction information, $1.765 \AA$ and $1.802 \AA$ are the reported bond lengths. Thus, non-covalent interactions have a crucial impact on bond length of covalent bonds; in particular, we have focused on the C-S bond, which links N3 with the main protease that is an important bond in these systems. Naturally, there are other effects that affect C-S bond lengths (protein packing, for example). However, from here it is clear that non-covalent interactions impact also in the description of covalent bonds. Therefore, it is important to conclude that the description of all hydrogen bonds involved in the inhibitor-protease interaction (conventional and non-conventional) is necessary to fully understand the possible action mechanism of these drugs.

\section{Methods}

Crystal structures of the main protease of SAR-CoV (SARS-CoV M ${ }^{\text {pro }}$ ) and SAR-CoV-2 (SARS-CoV-2 M ${ }^{\text {pro }}$ ) viruses in complex with the peptide inhibitor N3 have been reported recently (PDB ID 2AMQ and 6LU7, respectively) ${ }^{5,6}$. It is important to mention that the $\mathrm{N} 3$ inhibitor is covalently bounded to $\mathrm{M}^{\text {pro }}$ through a C-S bond between the CYS144 of $\mathrm{M}^{\text {pro }}$ and the PJE of N3. To identify and quantify intermolecular and intramolecular interactions between the N3 and the SARS-CoV $\mathrm{M}^{\text {pro }}$ and the SARS-CoV-2 $\mathrm{M}^{\text {pro }}$, in this article several molecular systems have been constructed from their respective X-ray crystallographic structures in the following way: a) water molecules were removed from structures, b) hydrogen atoms were added, c) ionic residues were protonated or deprotonated according to Protonate-3D application implemented in Molecular Operating Environment (MOE 2016.08; Chemical Computing Group, Inc., Montreal, QC, Canada). The difference between 
the systems are the contacts residues of the SARS-CoV $\mathrm{M}^{\text {pro }}$ or SARS-CoV-2 $\mathrm{M}^{\text {pro }}$ respect to N3 molecule. Then, for the N3-SARS-CoV M $\mathrm{M}^{\text {pro }}$ and N3-SARS-CoV-2 $\mathrm{M}^{\text {pro }}$ models, a cutoff radius of $4.0 \AA$ A was applied. That is, we consider any $\mathrm{M}^{\text {pro }}$ residue with at least one atom up to a distance of $4.0 \AA$ respect to any atom of N3. Each of these two systems contains 469 atoms. The cavities provided by the $\mathrm{M}^{\text {pro }}$ models are depicted through the molecular electrostatic potential in Figure 3. In addition, we have considered one system containing the N3 inhibitor covalently bounded to the CYS144 (111 atoms), and other system considering a cutoff radius of $4.0 \AA$ but using the PJE residue of the N3 inhibitor as a reference (230 atoms). These two systems were also built from the N3-SARS-CoV-2 $\mathrm{M}^{\text {pro }}$ complex. In all generated systems, the different peptide fragments were capped at the $\mathrm{N}$ - and C-termini in the neutral states or methylated. All these structures are included in the ESI. The electronic structure of all systems was obtained by using the PBE0-D3/6-311G(d,p $)^{16,17}$ method with the Terachem code ${ }^{18}$. The molecular electrostatic potential ${ }^{19}$, NCI and QTAIM analysis were obtained by the graphics processing units for atoms and molecules (GPUAM) project ${ }^{12,20}$, developed in our group.

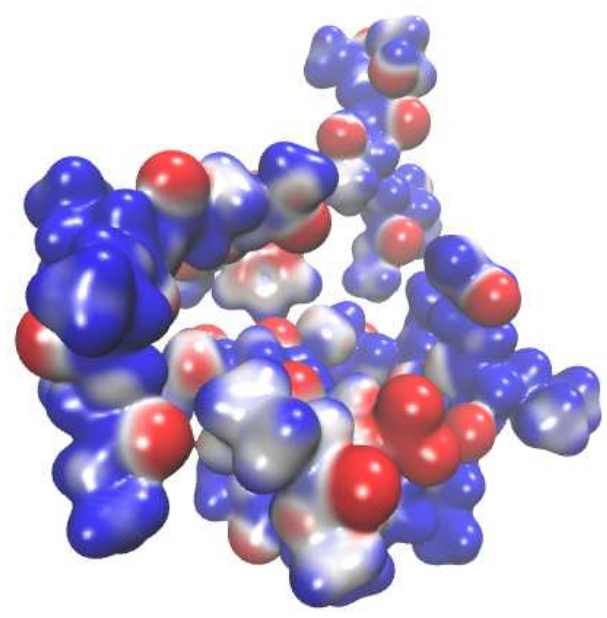

(a)

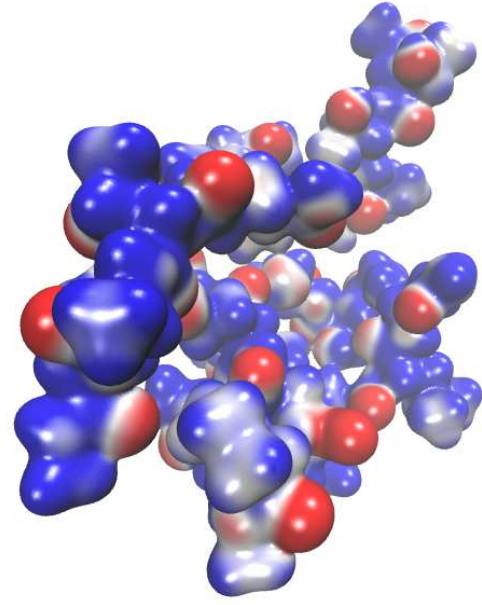

(b)

Figure 3. Molecular electrostatic potential of SARS-CoV M $\mathrm{M}^{\text {pro }}$ (a) and SARS-CoV-2 $\mathrm{M}^{\text {pro }}$ (b) models used in this article.

\section{References}

1. Menachery, V. D. et al. A SARS-like cluster of circulating bat coronaviruses shows potential for human emergence. Nat. Med. 21, 1508-1513 (2015).

2. South, A. M., Tomlinson, L., Edmonston, D., Hiremath, S. \& Sparks, M. A. Controversies of renin-angiotensin system inhibition during the COVID-19 pandemic. Nat. Rev. Nephrol. DOI: 10.1038/s41581-020-0279-4 (2020).

3. Wang, F., Chen, C., Tan, W., Yang, K. \& Yang, H. Structure of Main Protease from Human Coronavirus NL63: Insights for Wide Spectrum Anti-Coronavirus Drug Design. Sci. Rep. 6, 22677 (2016).

4. Bosch, B., van der Zee, R., de Haan, C. \& Rottier, P. The coronavirus spike protein is a class I virus fusion protein: Structural and functional characterization of the fusion core complex. J. Virol. 77, 8801-8811 (2003).

5. Jin, Z. et al. Structure of Mpro from SARS-CoV-2 and discovery of its inhibitors. Nature DOI: 10.1038/s41586-020-2223-y (2020).

6. Yang, H. et al. Design of wide-spectrum inhibitors targeting coronavirus main proteases. PLoS. Biol. 3, 1742-1752 (2005).

7. Anand, K., Ziebuhr, J., Wadhwani, P., Mesters, J. \& Hilgenfeld, R. Coronavirus main proteinase (3CL(pro)) structure: Basis for design of anti-SARS drugs. Science 300, 1763-1767 (2003).

8. Yang, H. et al. The crystal structures of severe acute respiratory syndrome virus main protease and its complex with an inhibitor. Proc. Natl. Acad. Sci. U. S. A. 100, 13190-13195 (2003).

9. Hatada, R. et al. Fragment Molecular Orbital Based Interaction Analyses on COVID-19 Main Protease - Inhibitor N3 Complex (PDB ID:6LU7). ChemRxiv DOI: 10.26434/chemrxiv.11988120.v1 (2020).

10. Bader, R. F. W. Atoms in molecules: A quantum theory. International series of monographs on chemistry (Clarendon Press, 1990). 
11. Johnson, E. R. et al. Revealing noncovalent interactions. J. Am. Chem. Soc. 132, 6498-6506 (2010).

12. Hernández-Esparza, R., Vázquez-Mayagoitia, A., Soriano-Agueda, L. A., Vargas, R. \& Garza, J. GPUs as boosters to analyze scalar and vector fields in quantum chemistry. Int. J. Quantum Chem. 119, e25671 (2019).

13. Vargas, R., Garza, J., Dixon, D. \& Hay, B. How strong is the C-alpha-H center dot center dot center dot $\mathrm{O}=\mathrm{C}$ hydrogen bond? J. Am. Chem. Soc. 122, 4750-4755 (2000).

14. Yang, L., Hubbard, T. A. \& Cockroft, S. L. Can non-polar hydrogen atoms accept hydrogen bonds? Chem. Commun. 50, 5212-5214 (2014).

15. Espinosa, E., Molins, E. \& Lecomte, C. Hydrogen bond strengths revealed by topological analyses of experimentally observed electron densities. Chem. Phys. Lett. 285, 170-173 (1998).

16. Adamo, C. \& Barone, V. Toward reliable density functional methods without adjustable parameters: The pbe0 model. $J$. Chem. Phys. 110, 6158-6170 (1999).

17. Francl, M. M. et al. Self-consistent molecular orbital methods. xxiii. a polarization-type basis set for second-row elements. J. Chem. Phys. 77, 3654-3665 (1982).

18. Ufimtsev, I. S. \& Martinez, T. J. Quantum chemistry on graphical processing units. 3. analytical energy gradients, geometry optimization, and first principles molecular dynamics. J. Chem. Theory Comput. 5, 2619-2628 (2009).

19. Cruz, J. C., Hernández-Esparza, R., Vázquez-Mayagoitia, A., Vargas, R. \& Garza, J. Implementation of the Molecular Electrostatic Potential over Graphics Processing Units. J. Chem Inf. Model. 59, 3120-3127 (2019).

20. Hernández-Esparza, R. et al. Grid-based algorithm to search critical points, in the electron density, accelerated by graphics processing units. J. Comput. Chem. 35, 2272-2278 (2014).

\section{Acknowledgements}

This work was supported by CONACYT, México, through the project FC- 2016/2412. The authors thank the facilities provided by the Laboratorio de Supercómputo y Visualización en Paralelo at the Universidad Autónoma Metropolitana -Iztapalapa.

\section{Author contributions statement}

P. G.-G. and R. A. Z. analyzed and built the molecular structures from X-ray data. R. V. and J. G. performed the quantum chemistry calculations. A. M., I. A. I., R. V. and J. G. wrote the main structure of the manuscript. All authors contributed and reviewed the manuscript.

\section{Additional information}

Electronic Supporting Information accompanies this paper.

The author(s) declare no competing interests. 
Figures



(I)

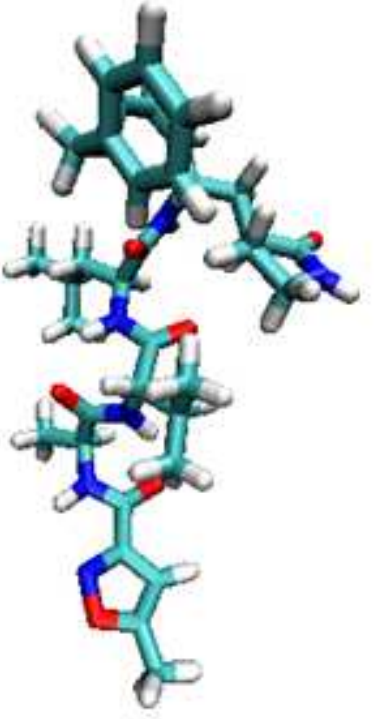

(II)

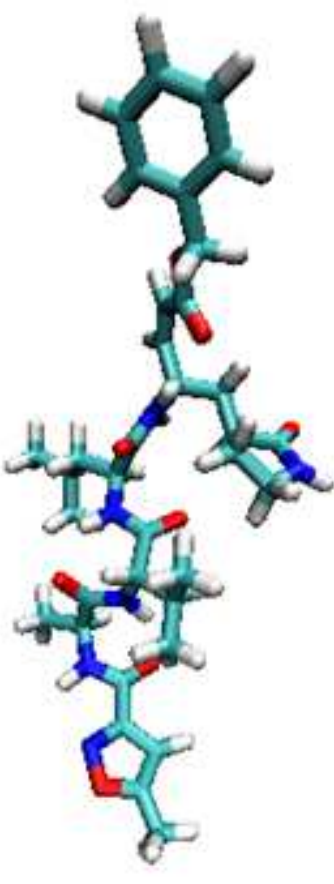

(III)

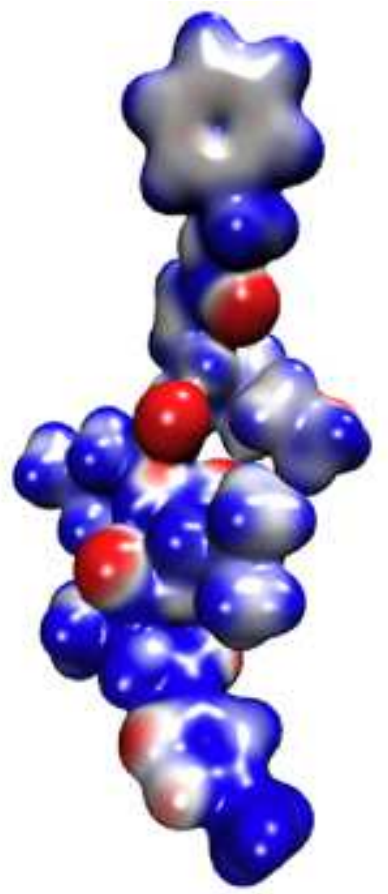

(MEP of III)

\section{Figure 1}

Three rotamers of the N3 inhibitor. The Molecular Electrostatic Potential (MEP) of the rotamer (III) is depicted on the right side as an example. Blue regions indicate positive values and red zones indicate negative values. 


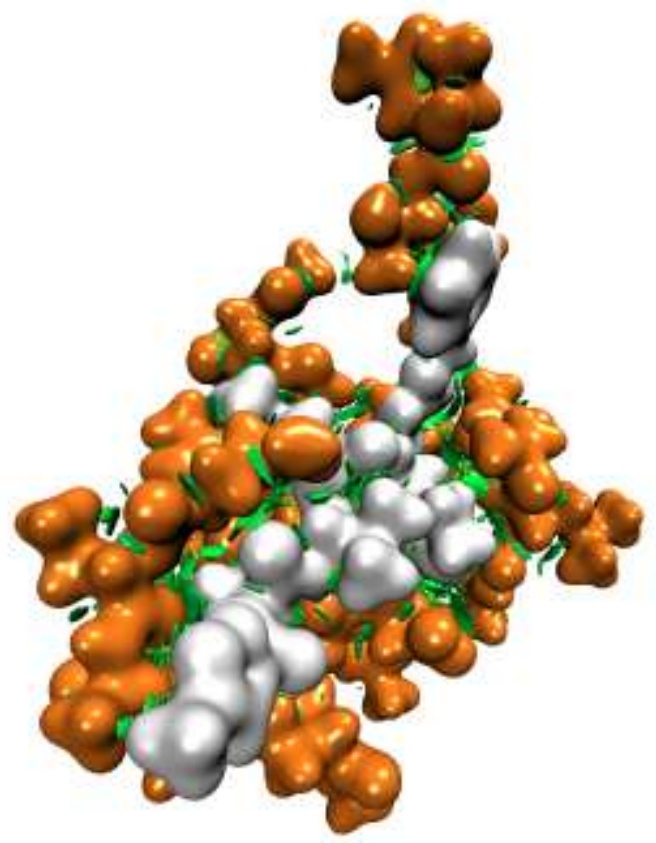

(a)

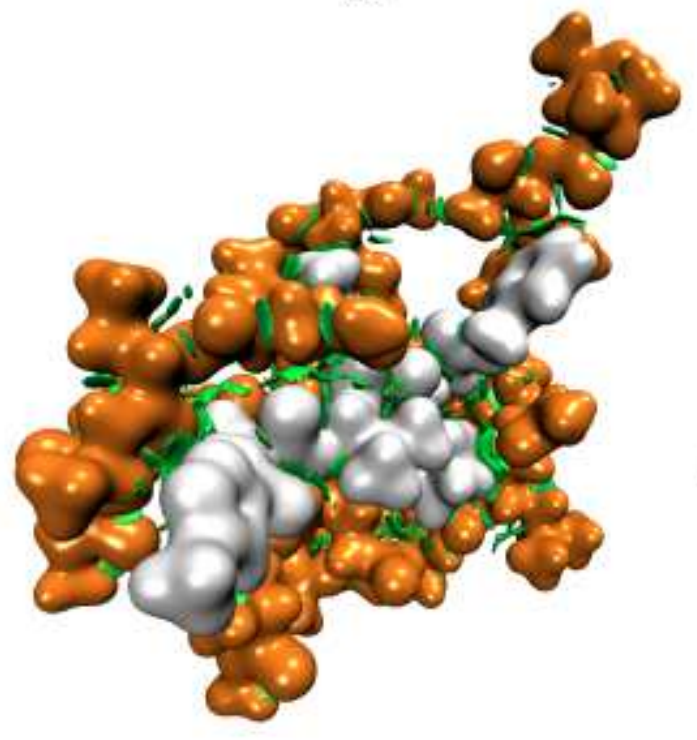

(c)

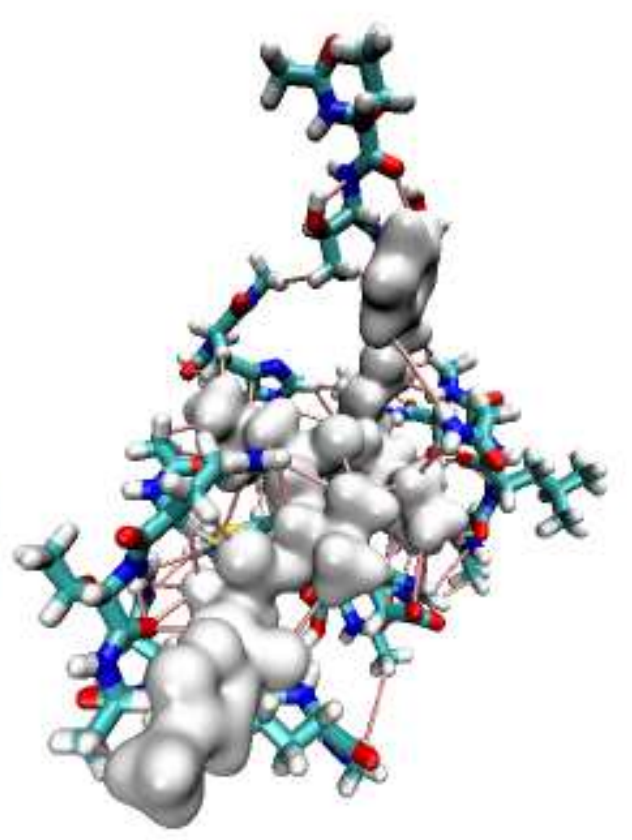

(b)

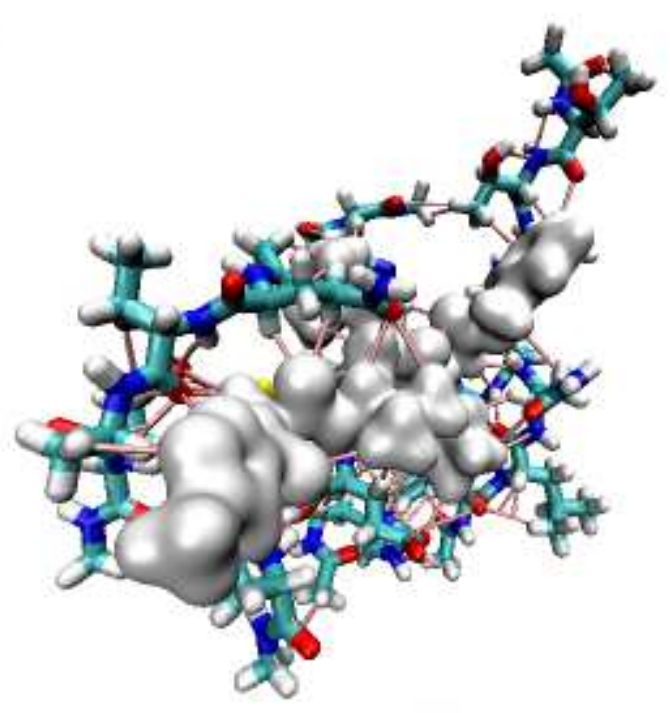

(d)

\section{Figure 2}

Isosurfaces of the electron density (orange for protase and white for inhibitor) and non-colvalent interaction index (green) for N3-SARS-CoV Mpro (a) and N3-COVID-19 Mpro (c)complexes. Inter- and intra-molecular contacts depicted through bond paths (pinks) provided by the QTAIM for N3-SARS-Cov Mpro (b) and N3-SARS-CoV-2 Mpro (d) systems. 


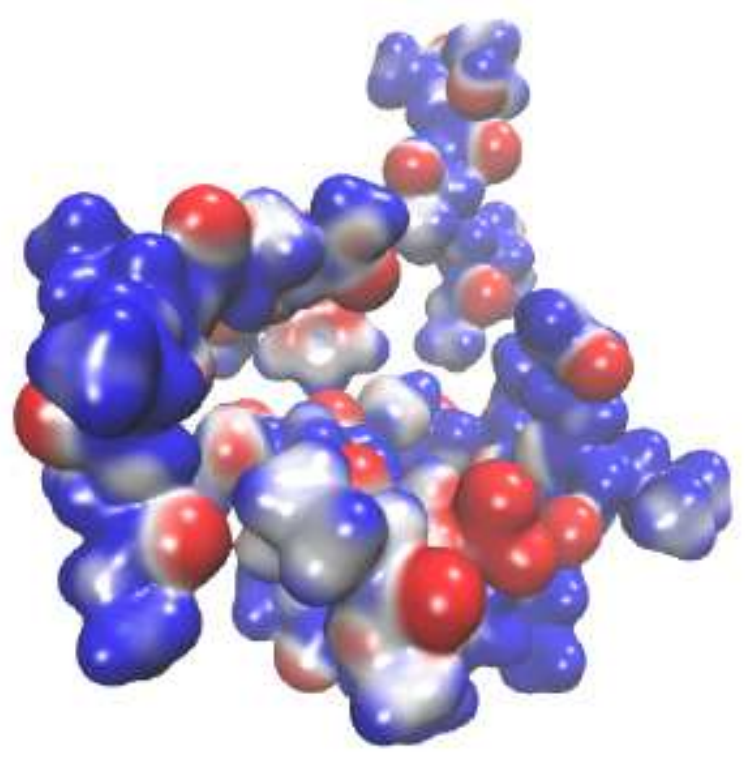

(a)

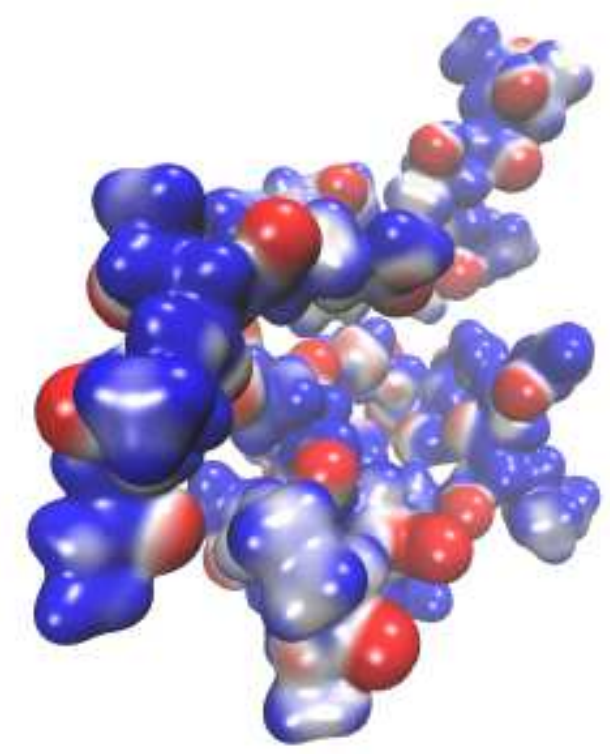

(b)

Figure 3

Molecular electrostatic potential of SARS-CoV Mpro (a) and SARS-CoV-2 Mpro (b) models used in this article.

\section{Supplementary Files}

This is a list of supplementary files associated with this preprint. Click to download.

- ESIcovid19.pdf 